\title{
Variational Principle for Self-replicating Systems
}

\author{
O. Kuzenkov*, E. Ryabova \\ Lobachevsky State University of Nizhni Novgorod
}

\begin{abstract}
The biological theory of natural selection is the key idea for understanding optimality in biology. Selection processes are the base of variational principles in modern biological theory. Biological variational principles are most justified when they are the consequence of selection processes. But the use of the variational principles for explaining strategies of behavior of living species is a difficult problem. In this paper, an order of preference is introduced on the set of hereditary strategies of behavior in general self-replicating systems as a result of selection.

The introduced order of preference is expressed with the help of the comparison criterion, which is an optimality criterion in self-replicating systems. The comparison is made between all kinds of continuous functions of behavior rather than between some discrete collections of variations. Maximization of this criterion is a variational principle in general self-replicating systems.

The newly introduced selection criterion has a series of peculiarities, which are analyzed in this article. One of them is the outcome dependence on initial conditions; in particular the criterion value does not satisfy transitivity while changing the initial conditions. The second feature is the result of the velocity dependence of transients during adaptation. Besides, sometimes the best strategy from the standpoint of the criterion can lead to the system extinction. Methods of accounting of these peculiarities are proposed for optimization of self-replicating systems.
\end{abstract}

Keywords and phrases: optimality, selection, optimization criterion, order of preference

Mathematics Subject Classification: 93A30, 91B08, 92D15

\section{Introduction}

Darwin's theory, which was formed in the 19th century, gave an explanation of the amazing adaptability of living things [11]. The biological theory of natural selection is the key idea for understanding optimality in biology.

The development of a mathematical model of selection in biology has continued since the appearance of the basic works of Haldane [21] in the 1920s. At the same time, these models have been considered by R. Fisher [15] and S. Wright [59]. Selection phenomena have been discovered in different mathematical models of population dynamics, ecological systems and genetic processes $[3,5,7,45,47,50,53]$.

The selection processes are of paramount importance, not only in biology. Generally selection is the process of a progressive reduction of the initial set of similar elements to a smaller subset by excepting a part of elements (in accordance with some criterion). Various aspects of selection in mathematical models

${ }^{*}$ Corresponding author. E-mail: kuzenkov_o@mail.ru 
of self-replicating systems were investigated by L. Rozonoer and E. Sedyh [49]. In the 1980s A. Gorban created a general mathematical theory of selection [18-20]. He developed a universal mathematical tool for modelling selection processes, established the fundamental facts of selection, in particular, the selection theorem.

Selection processes are the base of variational principles in modern biological theory. Nowadays variational principles are frequently used for mathematical modeling of biological phenomena $[24,39,42,51,62]$ (in the same way as they are used in mechanics and optics). However, variational principles implemented in biological evolution are often based on conventional wisdom and lack of mathematical justification. Today more attention is devoted to justification of those principles $[27,30]$. To formulate a variational principle, it is necessary to determine the criterion or comparison functional. This criterion of comparison can be expressed correctly on the basis of Darwin's principle of natural selection (survival of the fittest). That is why variational principles are most justified when they are the consequence of selection processes.

Different optimality principles of biological systems were formulated on the basis of selection $[8,19,20$, $26,46,48]$. There is a maximum principle of the time average value of the reproduction coefficient $[15,18$ $21,35-37,50,53,59]$, as well as of life reproductive success of individuals [55,56] and offspring biomass [25], survival [22,23], reproductive effort [52,63], lifetime fitness function [9], etc.

In the simplest case, the variational principles can be applied to model evolution of phenotypes, but their use for explaining strategies of behavior of living species is more difficult problem. In this paper, an order of preference is introduced on the set of hereditary strategies of behavior in general self-replicating systems as a result of selection. The comparison is made between all kinds of continuous functions of behavior rather than between some discrete collections of variations. The introduced order is expressed mathematically with the help of a certain functional, whose maximization is a variational principle in general self-replicating systems. Based on this principle, the problem of calculus of variations for determining the mode of daily vertical migrations of zooplankton as a periodical continuous function of time is solved.

The newly introduced selection criterion has a series of peculiarities such as the outcome dependence on initial conditions, in particular the criterion value does not satisfy transitivity while changing the initial conditions, and the outcome dependence on the rate of transients at adaptation. Besides, sometimes the best strategy from the standpoint of the criterion can lead to the system extinction. Methods of accounting of these peculiarities are proposed for optimization of self-replicating systems.

The obtained results can be effectively used to processes control of self-reproduction and selection, for example, as it was done in $[33,37]$.

\section{Motivation example: Migrations of aquatic organisms}

In the beginning, consider the problem of diel vertical movements (DVM) of zooplankton as a motivation example.

A wide variety of marine and freshwater plankton organisms are subject to diel vertical migration, changing their vertical distribution in the water column in the $24 \mathrm{~h}$ cycle $[2,13,61]$. For example, the cladoceran Bosmina coregoni in Babine Lake (British Columbia) performs vertical migrations, rising to the surface during the day and descending to deeper water at night [40].

This behavior has been studied for more than a century. The relative complexity and diversity of vertical migration makes it difficult to find a unifying theory to explain the different migration patterns exhibited by zooplankton species. Several hypotheses, such as the predator-evasion hypothesis, foodavailability hypothesis, light-protection hypothesis have been proposed to explain its adaptive significance.

For example, the dynamic DVM model was constructed in the works of M. Mangel and C. Clark. The problem was considered by M. Mangel, C. Clark for discrete time [9]. Building this model is based on the idea of the expected intake rate maximization, in particular, on the hypothesis of lifetime fitness function maximization that is the maximal probability of the individual's survival in the time period given that at the initial time the individual is alive. 
In the present work the dynamic behavior model of zooplankton is constructed in continuous time. Moreover, the comparison is made between all kinds of continuous functions of behavior rather than between some discrete collections of variations.

It is known that the conditions of zooplankton existence depend on the depth of the water layer and change periodically throughout each day. A near-surface habitat of the cladoceran contains food (phytoplankton) and predators; a deep water habitat does not contain food and predators. The predators may be active during the day, or during the night, but not both. Instantaneous mortality risk for a zooplankter subject to predation is given in Figure 1.

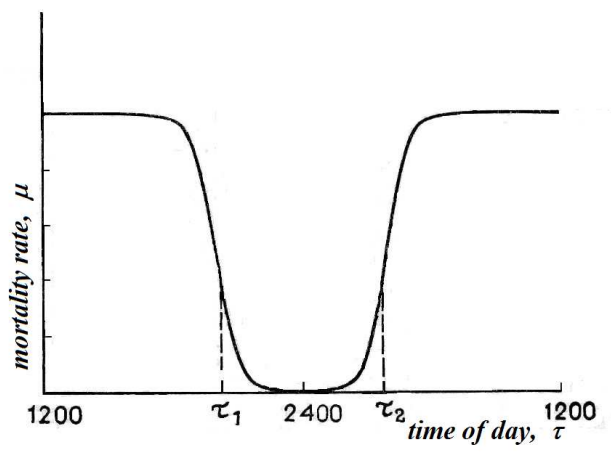

FigURE 1. Instantaneous mortality risk for a zooplankter subject to predation [9].

Let $z$ be the vertical coordinate of the water layer. The measurement scale of the vertical coordinate is chosen to possible zooplankton oscillations ranged from -1 to $1,-1 \leq z \leq 1$. We suppose that the individual fitness of zooplankton can depend on the value of $z$. Let $R$ be the maximum possible value of the individual fitness in the most favorable conditions in the absence of competition.

We assume that the coordinate of the most favorable level changes over time by law $z_{0}(t)=\sin (2 \pi t)$ (here the time $t$ is measured in days), the individual fitness for the layer $z$ decreases as the distance from the most favorable layer and is expressed as follows $R-\left(z-z_{0}(t)\right)^{2}$. Assume also that the metabolic costs are proportional to the square of the change speed of the vertical position: $\beta(\dot{z})^{2}$. Then the total value of the individual fitness $r$ is of the form

$$
r=\left(R-\left(z-z_{0}(t)\right)^{2}\right)-\beta(\dot{z})^{2} .
$$

The first term in (2.1) reflects the influence of the zooplankton position on its reproduction; the second term reflects the decrease of the reproduction because of the metabolic cost. At the initial time $t=0$ the coordinate $z(0)$ can have any value, but at the final time $t=1$ the coordinate $z(1)$ must coincide with $z(0)$, otherwise the function $z(t)$ cannot be continuous periodic. This leads to the equation $z(0)=z(1)$. If the speed of the vertical movement is known, the function $z(t)$ is uniquely determined by the given initial conditions $z(0)$.

Let the zooplankton population realize the strategy $z^{*}(t)$ of changing the vertical coordinate. Let $z^{*}(t)$ be a continuous periodic function of periods 1: $z^{*}(0)=z^{*}(1)$. Let $x^{*}(t)$ be the number of zooplankton in a population, which realizes the strategy $z^{*}$ at the moment $t ; r\left(z^{*}\right)$ is the fitness of zooplankton corresponding strategy $z^{*} ; r\left(z^{*}(t)\right)$ is a periodic time function too.

We suppose that a new type of behavior $z_{\epsilon}(t)$ has appeared in the zooplankton population as result of mutation or in some other way. Let $z_{\epsilon}(t)$ also be a continuous periodic function: $z_{\epsilon}(0)=z_{\epsilon}(1)$. In the theory of calculus of variations the function $z_{\epsilon}$ is called a variant. Let $x_{\epsilon}(t)$ be the number of zooplankton in a population, which realizes the strategy $z_{\epsilon}$ at the moment $t$; and $r\left(z_{\epsilon}\right)$ be the fitness of zooplankton corresponding strategy $z_{\epsilon}$; and $r\left(z_{\epsilon}(t)\right)$ be a periodic time function too. 
We suppose that strategies $z^{*}, z_{\epsilon}$ are genetically fixed; the population growth has the limitation proportional to the total number of zooplankton; then the equations for $x^{*}$ and $x_{\epsilon}$ take the form

$$
\dot{x}^{*}=r\left(z^{*}\right) x^{*}-x^{*}\left(x^{*}+x_{\epsilon}\right), \quad \dot{x}_{\epsilon}=r\left(z_{\epsilon}\right) x_{\epsilon}-x_{\epsilon}\left(x^{*}+x_{\epsilon}\right) .
$$

This system of replicator equations can also be considered as a generalization of the Verhulst's model of biomass growth when biomass is of heterogeneous components. In addition, it complies with the idea of natural selection from Dawkins' Universal Darwinism [12], the relative rate of change of the proportion of the type $z^{*}$ (respectively $z_{\epsilon}$ ) is the difference of the fitness of the type $z^{*}$ (respectively $z_{\epsilon}$ ) and the average population fitness.

It is easy to see that the relationship $x^{*} / x_{\epsilon}$ satisfies the equations

$$
\frac{d}{d t}\left(\frac{x^{*}}{x_{\epsilon}}\right)=\left(r\left(z^{*}\right)-r\left(z_{\epsilon}\right)\right) \frac{x^{*}}{x_{\epsilon}}
$$

Then

$$
\frac{x^{*}}{x_{\epsilon}}(t)=\frac{x^{*}}{x_{\epsilon}}\left(t_{0}\right) \exp \int_{t_{0}}^{t}\left(r\left(z^{*}\right)-r\left(z_{\epsilon}\right)\right) d t
$$

Using the periodicity functions $r$ (period is $t=1$ ), it can be seen that in the case of integer $t_{0}$ and $t$

$$
\int_{t_{0}}^{t}\left(r\left(z^{*}\right)-r\left(z_{\epsilon}\right)\right) d t=\left(t-t_{0}\right) \int_{t_{0}}^{t_{0}+1}\left(r\left(z^{*}\right)-r\left(z_{\epsilon}\right)\right) d t .
$$

If

$$
\int_{t_{0}}^{t_{0}+1} r\left(z^{*}\right) d t<\int_{t_{0}}^{t_{0}+1} r\left(z_{\epsilon}\right) d t
$$

then

$$
\lim _{t \rightarrow \infty} \frac{x^{*}}{x_{\epsilon}}(t)=0 \text { and } \lim _{t \rightarrow \infty} x^{*}(t)=\lim _{t \rightarrow \infty} x_{\epsilon}(t) \frac{x^{*}(t)}{x_{\epsilon}(t)}=0
$$

Thus, zooplankton realizing the strategy $z_{\epsilon}$ outcompetes zooplankton that realized the strategy $z^{*}$. Some time after completion of the transition processes the zooplankton population will not contain the plankton which realizes the strategy $z^{*}$. Consequently, the strategy $z^{*}$ will be present in the population over time only if it realizes the maximum of functional $\int_{t_{0}}^{t_{0}+1} r\left(z^{*}\right) d t$.

Hence, we can formulate the variational principle: the real strategy of zooplankton population is one that provides the maximum of functional $\int_{t_{0}+1}^{t_{0}+1} r\left(z^{*}\right) d t$. This variational principle is based on the selection process. The functional $\int_{t_{0}}^{t_{0}+1} r\left(z^{*}\right) d t$ is the optimality criterion of the objects' existence, because only objects with a maximum value of this functional remain in the system over time.

Using the periodicity conditions, the problem is reduced to finding the function $z^{*}(t)$, satisfying equality (2), for which the functional $\int_{0}^{1}\left(R-\left(z-z_{0}(t)\right)^{2}-\beta \dot{z}^{2}\right) d t$ takes its maximum value. This problem is a classic problem of calculus of variations [60]. By solving it, we find that optimal changes of the coordinate $z$ is of the form $z^{*}=\frac{1}{1+\beta} \sin t$.

If metabolic costs are large compared with the gains from changing location ( $\beta$ is large), then the vertical movements are negligible. If metabolic costs for changing positions are small compared to the gains ( $\beta$ is small), then the vertical movements are substantial. In particular, the optimal provision always coincides with the best layer when $\beta=0$.

The solution can be used to explain the real behavior of zooplankton [9]. 


\section{Optimality criterion for self-replicating systems}

We can obtain a similar variational principle for general self-replicating systems. Self-replicating objects are objects that can produce copies of themselves. The phenomena of self-reproduction are widespread in different areas: in biology, ecology, genetics, economics, etc. For example, live organisms are selfreplicating objects in biology. Classical models of population dynamics of Malthus, Verhulst, Volterra, Bazykin are self-replicating systems $[3,38,57,58]$. Mathematical properties of biological self-reproducing systems were considered by J. Haldane, R. Fisher, S. Wright $[15,21,59]$. J. Neumann studied selfreproducing objects in cybernetics [41]. Self-reproducing systems in the general form were investigated by L. Rosonoer and E. Sedykh [49]. A. Gorban introduced general systems with inheritance as equations of measure dynamics [19]. In recent years, models of self-replicating systems have been intensively studied $[4-6,10,14,24,28,29,36,37]$. Some aspects of self-replicating systems optimization were considered in works [33-37].

Let $v=\left\{v_{1}, \ldots, v_{n}\right\}$ be the set of possible strategies for the behavior of the self-reproducing objects. Let $x_{i}$ be the number (or proportion) of the type $i$ in the self-replicating system that realized the strategy $v_{i}$ at the moment $t, x=\left(x_{1}, \ldots, x_{n}\right)$, and $k_{i}(x, t)$ be the relative rate of change of the objects' number (or proportion) of the $i$-th type. Then $k_{i}(x, t)$ can be called a natural increase of the self-reproducing objects of $i$-th type as well as in demographics. And $k_{i}(x, t)$ can also be called the reproduction rate. Then $x_{i}$ satisfy the equation system

$$
\dot{x}_{i}=k_{i}(x, t) x_{i}, \quad i=\overline{1, n} .
$$

Suppose we are given the initial state of the system $x\left(t_{0}\right): x_{i}\left(t_{0}\right)>0, i=\overline{1, n}$.

We can compare the self-reproducing objects with different strategies based on the mathematical theory of selection.

Definition 3.1. We say that objects of $j$-th type are better than the objects of $i$-th type or the strategy $v_{j}$ is better than $v_{i}\left(v_{j} \succ v_{i}\right)$, if

$$
\lim _{t \rightarrow \infty} \frac{x_{i}(t)}{x_{j}(t)}=0
$$

Thus, the order of preference is introduced on the set of different self-reproducing objects. In this case objects of the $j$-th type outcompete objects of the $i$-th type.

Assume that we have an upper bound for the total number of all objects $X(t)=\sum_{i=1}^{n} x_{i}(t) \leq W$, where $W$ - is a constant; $0<X\left(t_{0}\right)<W$; and objects of the $j$-th type outcompete objects of the $i$-th type. Then the number of objects of the $i$-th type tends to zero over time. Indeed, since $x_{i}=\frac{x_{j} x_{i}}{x_{j}} \leq W \frac{x_{i}}{x_{j}}$, we have $\lim _{t \rightarrow \infty} x_{i}=0$. Consequently, the $i$-th type gradually disappears. A species can persist for an unlimited amount of time only if all other species are subordinate to it relative to the introduced order.

It is advisable to express the entered order of preference through the comparison of some numerical values. The following propositions are valid provided that $0<x_{i}(t)<W, \quad i=\overline{1, n}$.

Criterion 3.2. Strategy $v_{j}$ is better than $v_{i}\left(v_{j} \succ v_{i}\right)$ iff the relation

$$
\lim _{t \rightarrow \infty} \int_{t_{0}}^{t}\left(k_{j}(x, t)-k_{i}(x, t)\right) d t=+\infty
$$

is true.

Proof. By (3.1), the fraction $\frac{x_{i}}{x_{j}}$ satisfies the differential equation

$$
\frac{d}{d t}\left(\frac{x_{i}}{x_{j}}\right)=\frac{x_{i}}{x_{j}}\left(k_{i}(x, t)-k_{j}(x, t)\right)
$$


and

$$
\frac{x_{i}}{x_{j}}(t)=\frac{x_{i}}{x_{j}}\left(t_{0}\right) \exp \left(-\int_{t_{0}}^{t}\left(k_{j}(x, t)-k_{i}(x, t)\right) d t\right) .
$$

Since $0<x_{i}(t)<1$ and $0<x_{j}(t)<1$ for all $t \geq t_{0}$, the fraction $\frac{x_{i}}{x_{j}}\left(t_{0}\right)$ is a bounded quantity. If (3.2) is true, then $\lim _{t \rightarrow \infty} \frac{x_{i}}{x_{j}}=0$. By Definition 3.1, the strategy $v_{j}$ is better than $v_{i}$.

Conversely, let the strategy $v_{j}$ be better than $v_{i}$. By Definition 3.1, $\lim _{t \rightarrow \infty} \frac{x_{i}}{x_{j}}=0$. According to (3.3) the relation $(3.2)$ is true, because $\frac{x_{i}}{x_{j}}\left(t_{0}\right)$ is bounded.

Corollary 3.3. $v_{j} \succ v_{i}$ for all $i=\overline{1, n}, i \neq j$, iff the inequality (3.2) holds for all $i=\overline{1, n}, \quad i \neq j$.

This follows from formula (3.3) which holds for all $i=\overline{1, n}, i \neq j$.

Definition 3.4. Let $\xi(t)$ be a continuous function. If the limit $\lim _{t \rightarrow \infty} \frac{1}{t} \int_{t_{0}}^{t} \xi(\tau) d \tau$ exists, then

$$
\langle\xi\rangle=\lim _{t \rightarrow \infty} \frac{1}{t} \int_{t_{0}}^{t} \xi(\tau) d \tau
$$

is called a time average value of the function $\xi(t)$.

Remark 3.5. If a continuous function $\xi(t)$ has a limit as $t \rightarrow \infty$, then its time average value coincides with this limit.

It is easy to see that $\langle\xi\rangle=\lim _{t \rightarrow \infty} \frac{\int_{t_{0}}^{t} \xi(\tau) d \tau}{t}=\lim _{t \rightarrow \infty} \xi(t)$, as it follows from the l'Hopital's rule.

Corollary 3.6. Let the time average values $\left\langle k_{i}\right\rangle,\left\langle k_{j}\right\rangle$ exist, and they are different for different strategies. Then $v_{j} \succ v_{i}$ iff an inequality relation

$$
\left\langle k_{j}\right\rangle>\left\langle k_{i}\right\rangle
$$

is true.

Proof. Let $\varepsilon=\left\langle k_{j}\right\rangle-\left\langle k_{i}\right\rangle$ and $\varepsilon>0$, so $\lim _{t \rightarrow \infty} \frac{1}{t} \int_{t_{0}}^{t}\left(k_{j}(x, t)-k_{i}(x, t)\right) d t=\varepsilon$. Therefore the relation $(3.2)$ is true, that is $v_{j} \succ v_{i}$.

Conversely, let the relationship $v_{j} \succ v_{i}$ hold. Assume that $\left\langle k_{j}\right\rangle\left\langle\left\langle k_{i}\right\rangle\right.$. As it has been proved above from this assumption, the relation $v_{i} \succ v_{j}$ is fulfilled, which contradicts the condition $v_{j} \succ v_{i}$.

Corollary 3.7. $v_{j} \succ v_{i}$ for all $i=\overline{1, n}, i \neq j$, iff the inequality (3.4) holds for all $i=\overline{1, n}, \quad i \neq j$.

Thus, we can formulate the variational principle.

Proposition 3.8 (Variational principle). Only the behavioral strategy that provides the maximum of time average of the objects' reproduction rate will be presented in the self-replicating system over time.

Then the time average of the objects' reproduction rate is the criterion of the objects' existence.

In many applications, equations (3.1) have the form

$$
\dot{x}_{i}=x_{i}\left(r_{i}(x, t)-\varphi(x, t)\right), \quad i=\overline{1, n}, \quad \varphi(x, t)=\sum_{j=1}^{n} x_{j} r_{j}(x, t),
$$


where $x_{i}$ is the proportion of the type $i$ in the population, and $x=\left(x_{1}, \ldots, x_{n}\right)$ is the vector of the distribution of types in the population, then $r_{i}(x, t)$ is the fitness of the type $i$ (which is dependent on the population and time), and $\phi(x, t)$ is the average population fitness (given by the weighted average of the fitness of the $n$ types in the population). Equation (3.5) is the replicator equation, it is one of the fundamental equations of evolutionary dynamics [4-6, 10, 14, 24, 28, 29, 31, 32, 36, 37, 45].

The replicator equation is defined on the simplex

$$
S_{n}=\left\{\left(x_{1}, \ldots, x_{n}\right): x_{i} \geq 0, i=\overline{1, n} \sum_{i=1}^{n} x_{i}=1 .\right\}
$$

The standard simplex $S_{n}$ is invariant under replicator dynamics: a trajectory which begins in the simplex never leaves the simplex $[31,32,45,54]$.

Suppose that the time average values $\left\langle r_{l}\right\rangle$ and $\left\langle k_{l}\right\rangle=\left\langle r_{l}-\varphi\right\rangle$ exist for $l=i, j$, and the relationship $\left\langle r_{i}\right\rangle \neq\left\langle r_{j}\right\rangle$ holds. We'll show that the time average of the objects' reproduction rate and the time average of the objects' fitness are equivalent from the viewpoint of the objects' comparison.

Corollary 3.9. $v_{j} \succ v_{i}$ iff the inequality

$$
\left\langle r_{j}\right\rangle>\left\langle r_{i}\right\rangle
$$

is true. The strategy $v_{j}$ is the best of the admissible strategies $v_{i}$ iff inequality (3.6) holds for all $i=\overline{1, n}, \quad i \neq j$.

Proof. The time average value $\langle\phi\rangle$ exists because the time average values $\left\langle k_{i}\right\rangle=\left\langle r_{i}-\phi\right\rangle$ and $\left\langle r_{i}\right\rangle$ exist. The time average value $\langle\phi\rangle$ is a constant value, therefore $\left\langle r_{i}\right\rangle=\left\langle k_{i}\right\rangle+\langle\phi\rangle$. If inequality (3.6) holds, then inequality (3.4) holds too; and vice versa. Therefore, Corollary 3.9 follows from Corollaries 3.6 and 3.7.

Thus, the time average of the objects' fitness is equivalent to the optimality criterion of the objects' existence.

Example 3.10. Consider a self-replicating system in the form (3.5) (for example, some populations of insect pollinators), where the fitness $r_{i}$ was equal to $r_{i}^{0}$ at the initial time $t_{0}$. Then existence conditions are changed. For example, if the nearest flowering field is mowed, then the existence conditions of the insect pollinators will change [16]. Let constant $b_{i}$ be the fitness of the objects of the type $i$ in new existence conditions. We assume that the change rate of the fitness $r_{i}$ is the difference of $b_{i}$ and $r_{i}$ :

$$
\dot{r}_{i}=b_{i}-r_{i}, \quad i=\overline{1, n} .
$$

Observe that system (3.5), (3.7) describes the adaptation process with a negative feedback.

The solutions of equations (3.7) have the form

$$
r_{i}(t)=b_{i}+\left(r_{i}^{0}-b_{i}\right) \exp (-t) .
$$

Since $\lim _{t \rightarrow \infty} r_{i}(t)=b_{i},\left\langle r_{i}\right\rangle=b_{i}$. By Corollary 3.9 and Definition 3.1 objects of the $j$-th type are better than objects of the $i$-th type $\left(v_{j} \succ v_{i}\right)$ if $b_{j}>b_{i}$. Thus, the constants $b_{i}$ reflect the objective possibilities of self-replicating objects in a new existence conditions, because only objects with a maximum value of $b_{i}$ remain in the system over time.

\section{Some peculiarities of the optimality criterion for self-replicating systems}

The optimality criterion for self-replicating systems has some peculiarities in comparison with the usual criteria in optimization problems. First of all, the criterion value depends on the initial conditions, as well as the rate of transients at adaptation. To see these peculiarities, consider a self-replicating system in the following example. 
Example 4.1. Consider a self-replicating system in the form (3.5), when $n=2$. The fitness $r_{i}$ was equal to $r_{i}^{0}$ at the initial time $t_{0}$. Suppose that the existence conditions of the population have changed. Also suppose that the rate of the change of values $r_{i}$ is proportional to $x_{i}$ in the system (3.5). This assumption is justified, for example, for populations of social insects. In this case the rate of adaptation depends on the obtained information about existence conditions, the amount of information depends on the number of explorers which is a fixed part of the population. If the number of the population is increasing then there is more information and the rate of change of the reproduction coefficient is increasing.

In this case system (3.5), (3.7) has the following form

$$
\left\{\begin{aligned}
\dot{x_{1}}= & x_{1}\left(1-x_{1}\right)\left(r_{1}-r_{2}\right), \\
\dot{r_{1}}= & x_{1}\left(b_{1}-r_{1}\right), \\
\dot{r_{2}}= & \left(1-x_{1}\right)\left(b_{2}-r_{2}\right), \\
& x_{2}=1-x_{1} .
\end{aligned}\right.
$$

It can be seen that the system has an invariant plane $r_{2}=b_{2}$. In fact, the last equation in the system (4.1) is an identity, if the equality $r_{2}=b_{2}$ is substituted. We obtain a system in the invariant plane below

$$
\left\{\begin{array}{l}
\dot{x_{1}}=x_{1}\left(1-x_{1}\right)\left(r_{1}-b_{2}\right), \\
\dot{r_{1}}=x_{1}\left(b_{1}-r_{1}\right)
\end{array}\right.
$$

It is easy to see that points with the coordinate $x_{1}=0$ and the point with the coordinates $x_{1}=1$, $r_{1}=b_{1}$ are equilibrium states of the system (4.2). Equilibria on the line $x_{1}=0$ are stable at $r_{1}<b_{2}$, and unstable at $r_{1}>b_{2}$. The graph of phase trajectories in the invariant plane can be constructed by using the method of isoclines (Figure 2).

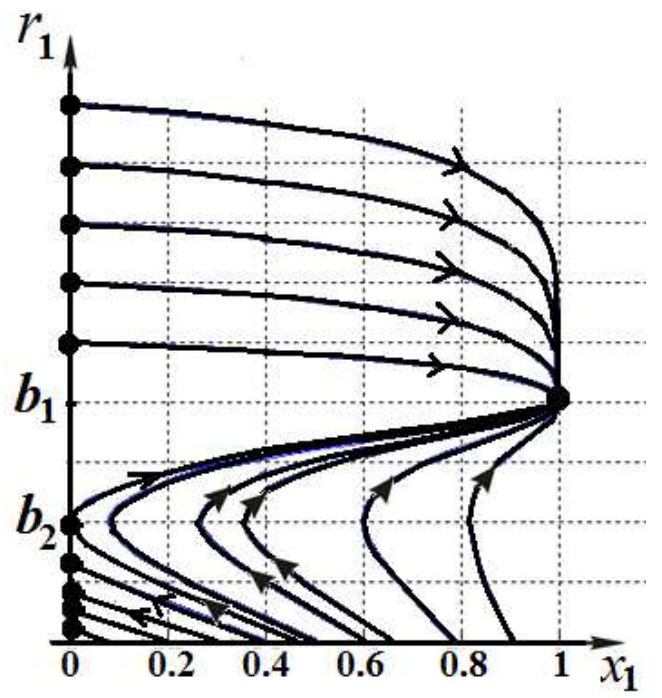

FiguRE 2. Graph of phase trajectories of system (4.1) in the invariant plane $r_{2}=b_{2}$.

The system dynamics is obvious to be the selection process with any initial condition. However, the selection result depends on the initial condition; and the best strategies are different for different initial conditions.

Figure 2 shows that the maximum possible value of $\left\langle r_{1}\right\rangle=b_{1}$ cannot be achieved under certain initial conditions $x_{1}\left(t_{0}\right)$. At these initial conditions the first species will be outcompeted from the system by the 
second kind of objects. The species with maximal $\langle r\rangle$ dies without having gone through the transition process. Species with the smallest time average of individual fitness survives, because the initial conditions are favorable to them and the transition process occurs quickly enough for these initial conditions. The species with the largest time average of the individual fitness can be outcompeted from the system, because it does not have time to adapt.

Thus, we have shown that the criterion value depends on the rate of transients during adaptation.

Since a value of the criterion depends on the initial conditions, the criterion value does not satisfy transitivity while changing the initial conditions. Besides this, sometimes the best strategy from the standpoint of the criterion can lead to the system extinction. To see these, consider a self-replicating system in the following example.

Example 4.2. Suppose that self-replicating objects can realize three behavior strategies in the system. Let it be $v_{1}, v_{2}$ and $v_{3}$. The strategy $v_{1}$ is the normal behavior of an individual, the strategy $v_{2}$ is the pathological behavior (illness, substance abuse, etc). The consequence of the pathological behavior strategy is the loss of the ability to reproduce. The strategy $v_{2}$ is not innate and can be transmitted to normal individuals (as a result of infection, imitation, copying, etc.). The probability of such transmission is proportional to the prevalence of this strategy in the system. The strategy $v_{3}$ is hereditarily assigned. Species realizing it are non-affected by disease (strategy $v_{2}$ ), but they have a smaller fitness $p$ in good conditions than species realizing strategy $v_{1}$. For example, it is known that the sickle cell anemia (strategy $v_{3}$ ) makes its carrier insensitive to malaria (strategy $v_{2}$ ) $[1,17]$.

Let $x_{1}, x_{2}, x_{3}$ be the numbers of individuals that realize the strategies $v_{1}, v_{2}, v_{3}$; values $x=\left(x_{1}, x_{2}, x_{3}\right)$ satisfy differential equations

$$
\begin{aligned}
& \dot{x}_{1}=a x_{1}-\frac{c x_{1} x_{2}}{x_{2}+x_{1}}-x_{1}\left(x_{1}+x_{2}+x_{3}\right), \\
& \dot{x}_{2}=\frac{c x_{1} x_{2}}{x_{2}+x_{1}}-a x_{2}-x_{2}\left(x_{1}+x_{2}+x_{3}\right), \\
& \dot{x}_{3}=p x_{3}-x_{3}\left(x_{1}+x_{2}+x_{3}\right),
\end{aligned}
$$

$0<p<a, c>2 a$. As we know $x_{1} \geq 0, x_{2} \geq 0, x_{3} \geq 0$ in this case [36].

Let $W=x_{1}+x_{2}+x_{3}$, then $\dot{W}=a x_{1}-a x_{2}+p x_{3}-W^{2}<a W-W^{2}$. If $W>a$, then $\dot{W}<0$ and $W$ decreases monotonically. Therefor $W$ is bounded by its initial value or the constant $a$. If $x_{1}(0)>0$, $x_{2}(0)>0, x_{3}(0)>0$, then the ratio $\frac{x_{1}}{x_{2}}$ satisfies the differential equation $\frac{d}{d t}\left(\frac{x_{1}}{x_{2}}\right)=\frac{x_{1}}{x_{2}}(2 a-c)$; the ratio $\frac{x_{2}}{x_{3}}$ satisfies the differential equation $\frac{d}{d t}\left(\frac{x_{2}}{x_{3}}\right)=\frac{x_{2}}{x_{3}}\left(\frac{c x_{1}}{x_{2}+x_{1}}-a-p\right)$. Therefor $\frac{x_{1}}{x_{2}}$ tends to zero, $x_{1}$ tends to zero, $\frac{x_{2}}{x_{3}}$ tends to zero, $x_{2}$ tends to zero. By Definition 3.1, $v_{3} \succ v_{2} \succ v_{1}$.

If $x_{1}(0)=0$, then $x_{1}(t)=0$,

$$
\begin{aligned}
& \dot{x}_{2}=-a x_{2}-x_{2}\left(x_{2}+x_{3}\right), \\
& \dot{x}_{3}=p x_{3}-x_{3}\left(x_{2}+x_{3}\right) .
\end{aligned}
$$

In this case the ratio $\frac{x_{2}}{x_{3}}$ satisfies the differential equation $\frac{d}{d t}\left(\frac{x_{2}}{x_{3}}\right)=\frac{x_{2}}{x_{3}}(-a-p) ;$ and $\frac{x_{2}}{x_{3}}$ tends to zero, $x_{2}$ tends to zero, $x_{3}$ tends to $p$. By Definition 3.1, $v_{3} \succ v_{2}$. This fact can also be ascertained with the help of Corollary 3.6. Since $\left\langle\dot{x}_{2} / x_{2}\right\rangle=\left\langle k_{2}\right\rangle=\lim _{t \rightarrow \infty}\left(-a-\left(x_{2}(t)+x_{3}(t)\right)=-a\right.$ and $\left\langle\dot{x}_{3} / x_{3}\right\rangle=\left\langle k_{3}\right\rangle=$ $=\lim _{t \rightarrow \infty}\left(p-\left(x_{2}(t)+x_{3}(t)\right)\right)=0,\left\langle k_{2}\right\rangle<\left\langle k_{3}\right\rangle$. By Corollary 3.6, $v_{3} \succ v_{2}$.

If $x_{2}(0)=0$, then $x_{2}(t)=0$,

$$
\begin{aligned}
& \dot{x}_{1}=a x_{1}-x_{1}\left(x_{1}+x_{3}\right), \\
& \dot{x}_{3}=p x_{3}-x_{3}\left(x_{1}+x_{3}\right) .
\end{aligned}
$$


In this case the ratio $\frac{x_{3}}{x_{1}}$ satisfies the differential equation $\frac{d}{d t}\left(\frac{x_{3}}{x_{1}}\right)=\frac{x_{3}}{x_{1}}(p-a) ;$ and $\frac{x_{3}}{x_{1}}$ tends to zero, $x_{3}$ tends to zero, $x_{1}$ tends to $a$. By Definition 3.1, $v_{1} \succ v_{3}$. Let us also confirm this result by using Corollary 3.6. Since $\left\langle\dot{x}_{1} / x_{1}\right\rangle=\left\langle k_{1}\right\rangle=\lim _{t \rightarrow \infty}\left(a-\left(x_{1}(t)+x_{3}(t)\right)=0\right.$ and $\left\langle\dot{x}_{3} / x_{3}\right\rangle=\left\langle k_{3}\right\rangle=$ $=\lim _{t \rightarrow \infty}\left(p-\left(x_{1}(t)+x_{3}(t)\right)\right)=-p-a<0,\left\langle k_{3}\right\rangle<\left\langle k_{1}\right\rangle$. By Corollary 3.6, $v_{1} \succ v_{3}$.

If $x_{3}(0)=0$, then $x_{3}(t)=0$, and we have system

$$
\begin{aligned}
& \dot{x}_{1}=a x_{1}-\frac{c x_{1} x_{2}}{x_{2}+x_{1}}-x_{1}\left(x_{1}+x_{2}\right), \\
& \dot{x}_{2}=\frac{c x_{1} x_{2}}{x_{2}+x_{1}}-a x_{2}-x_{2}\left(x_{1}+x_{2}\right) .
\end{aligned}
$$

Let $y=\frac{x_{1}}{x_{2}+x_{1}}$ be the proportion of the first type in the system, let $w=x_{1}+x_{2}$ be the total number of the system. Then $x_{1}=y w, x_{2}=(1-y) w$,

$$
\begin{aligned}
\dot{w} & =a w(2 y-1)-w^{2}, \\
\dot{y} & =(2 a-c) y(1-y) .
\end{aligned}
$$

Since $2 a<c, y \rightarrow 0$ and $w \rightarrow 0$. In this case the ratio $\frac{x_{1}}{x_{2}}=\frac{y}{1-y}$ tends to zero. By Definition 3.1, $v_{2} \succ v_{1}$. Moreover, $\left\langle\dot{x}_{1} / x_{1}\right\rangle=\left\langle k_{1}\right\rangle=\lim _{t \rightarrow \infty}(a-c(1-y)-w)=a-c,\left\langle\dot{x}_{2} / x_{2}\right\rangle=\left\langle k_{2}\right\rangle=\lim _{t \rightarrow \infty}(c y-a-w)=-a$. Since $a-c<-a$, we have $\left\langle k_{1}\right\rangle<\left\langle k_{2}\right\rangle$. By Corollary 3.6, $v_{2} \succ v_{1}$. Since the total number of the system tends to zero in this case, the strategy $v_{2}$ leads to the destruction of the system.

Thus, we have shown that the criterion value does not satisfy transitivity while changing the initial conditions. Also, sometimes the best strategy from the standpoint of the criterion can lead to the system extinction. By the way, in some other models effects of evolutionary extinction have been reported $[43,44]$.

Remark 4.3. The competitive displacement of all behavioral strategies except one, takes place only when the objects are pure lines. In fact, the population can persist all kinds of behavioral strategies by crossover and mutation, although the proportion of one strategy may be significantly higher than of all the others. This process is close to the selection process [37]. In this case, the behavioral strategies will be incomparable in terms of the introduced order of preference.

\section{Account of the peculiarities of the optimality criterion for modeling}

Let us show how the listed peculiarities of criterion can be taken into account in modeling so to get rid of unwanted effects generated by them. So, with the proper choice of the adaptation speed the order of preference of behavior strategies does not depend on the initial conditions.

Consider a self-replicating system in the form (3.5). Let the fitness $r_{i}$ be equal to constant $a_{i}^{0}$ at the initial time $t_{0}=1$. Suppose that the existence conditions of the population have changed. Also suppose that individual fitness $r_{i}$ changes as follows: $r_{i}(t)=\frac{a_{i}(t)}{t}, a_{i}(1)=a_{i}^{0}, i=\overline{1, n}$. The function $a_{i}$ changes over time and approaches a new value $b_{i}$ corresponding to new circumstances. This means that the adaptive effect is slowing. We assume that the changing rate of $a_{i}$ is proportional to $x_{i}$. Then the mathematical model of the self-replicating system has the form

$$
\left\{\begin{array}{l}
\dot{x}_{i}=\frac{a_{i}}{t} x_{i}-x_{i} \sum_{j=1}^{n} \frac{a_{j}}{t} x_{j}, \\
\dot{a}_{i}=x_{i}\left(b_{i}-a_{i}\right), i=\overline{1, n}, t \geq t_{0}=1 .
\end{array}\right.
$$

Theorem 5.1. If the inequalities $\left|a_{i}^{0}-a_{j}^{0}\right|<1-\varepsilon,\left|b_{i}-b_{j}\right|<1-\varepsilon,\left|a_{i}^{0}-b_{j}\right|<1-\varepsilon,\left|a_{j}^{0}-b_{i}\right|<1-\varepsilon$, $b_{i}>\max _{j=\overline{1, n}, j \neq i}\left\{b_{j}\right\}$ hold, then strategy $v_{i}$ is the best strategy from the admissible strategies $v_{j}$ for any initial conditions. 
The proof of Theorem 5.1 is given in Appendix.

If the conditions of Theorem 5.1 hold for the system (5.1), the best strategy from the standpoint of the optimality criterion will be the one that provides maximum fitness in the new (modified) circumstances. Thus, to eliminate the unwanted effects generated by peculiarities of criterion such as dependence on the initial conditions and the rate of transition processes during adaptation, it is necessary to influence (control) on the system to reduce the speed of adaptation. This fact can be used effectively in the construction of adaptive systems.

Now let us discuss how it is possible in modeling to avoid the loss of the system extinction.

If the total number of objects in the self-reproduction system cannot be less than some minimum positive value in any way of behavior, it means that the system always exists, regardless of the behavior. In this case, the optimization of the objects behavior from the standpoint of the system existence is meaningless.

The optimization of the objects' behavior makes sense for the system only in the case when one behavior leads the system to the destruction, but the other behavior supports its existence. So we have to assume that the number of objects in the system can be arbitrarily small.

From the assumptions it should be possible for an individual object to displace all others, but to destroy the entire system, just as unlimited growth of cancer cells leads to the destruction of the whole organism. D. Chernavsky [7] called this behavior "populism". In the interest of a self-preservation system it must have the mechanisms of confrontation the "populism".

Let $W$ be a maximal value of self-replicating objects in the system $x_{1}+x_{2}+\ldots+x_{n}<W$. Then $x_{n+1}=W-\left(x_{1}+x_{2}+\ldots+x_{n}\right)$ is the index of the system extinction $\left(v_{n+1}\right.$ can be called "strategy" of the system extinction). The strategy $v_{n+1}$ can be compared with the other $v_{i}$ based on the content of the Section 3: the strategy $v_{n+1}$ is better than $v_{i}\left(v_{n+1} \succ v_{i}\right)$ if $\lim _{t \rightarrow \infty}\left(x_{i} / x_{n+1}\right)=0$. Thus, the best strategy of the system behavior is not the one that supersedes all others, but the one that outcompetes the strategy of extinction. The system (4.4) does not have the best strategy of behavior; the "best" strategy is the strategy of extinction. The above proposed approach allows to distinguish "case" populism.

\section{Summary}

In this paper, the order of preference has been introduced on the set of hereditary strategies of behavior in general self-replicating systems as a result of selection. The introduced order has been expressed mathematically with the help of a certain functional. Thus optimality criterion has been expressed through selection processes. The variational principle has been formulated for general self-replicating systems as the maximization of this functional. The principle has been used to explain the observed biological phenomena. Based on this principle, the problem of calculus of variations for determining the mode of daily vertical migrations of zooplankton as a periodical continuous function of time has been solved. Properties of this criterion have been analyzed. Some of its peculiarities have been found. Ways of accounting of these peculiarities have been proposed.

The proposed variational principle can be used for the construction and study of behaviors of real biological systems, as well as for the control of their behavior. Obtained results can be effectively used in processes control of self-reproduction and selection, for example, as it was done in [33,37]. In particular, this principle can be used to solve the actual problem of modeling and explaining the phenomenon of diel vertical movements of zooplankton. Consideration of criterion peculiarities can be used to form effective control of adaptive systems and the development of mathematical numerical methods of adaptation. 


\section{Appendix}

Proof of Theorem 5.1. According to Corollary 3.3 strategy $v_{i}$ is the best strategy from the admissible strategies $v_{j}$ iff conditions

$$
\lim _{T \rightarrow \infty} \int_{t_{0}}^{T} \frac{a_{i}-a_{j}}{t} d t=+\infty \quad j=\overline{1, n}, \quad j \neq i,
$$

are fulfilled.

It is easy to see that the functions $a_{i}$ satisfy the following system of differential equations

$$
\frac{d}{d t}\left|b_{i}-a_{i}\right|=-x_{i}\left|b_{i}-a_{i}\right|, \quad i=\overline{1, n} .
$$

Since $\frac{d}{d t}\left|b_{i}-a_{i}\right|<0$, the value $\left|b_{i}-a_{i}\right|$ is decreasing; therefore, the following cases are possible for $b_{i}>b_{j}$ and different initial conditions $a_{i}^{0}, a_{j}^{0}$ :

1) if $a_{i}^{0}>b_{i}, a_{j}^{0}<b_{j}$, then $\left|a_{i}-a_{j}\right|<\left|a_{i}^{0}-a_{j}^{0}\right|<1-\varepsilon$;

2) if $a_{i}^{0}<b_{i}, a_{j}^{0}>b_{j}$, then $\left|a_{i}-a_{j}\right|<\left|b_{i}-b_{j}\right|<1-\varepsilon$;

3) if $a_{i}^{0}>b_{i}, a_{j}^{0}>b_{j}$, then $\left|a_{i}-a_{j}\right|<\left|a_{i}^{0}-b_{j}\right|<1-\varepsilon$;

4) if $a_{i}^{0}<b_{i}, a_{j}^{0}<b_{j}$, then $\left|a_{i}-a_{j}\right|<\left|a_{j}^{0}-b_{i}\right|<1-\varepsilon$.

Hence, the value $\left|a_{i}-a_{j}\right|$ satisfies the following inequalities

$$
\left|a_{i}-a_{j}\right|<1-\varepsilon \quad j=\overline{1, n}, \quad j \neq i .
$$

By (A-2) we have $\left|b_{i}-a_{i}\right|=\left|b_{i}-a_{i}^{0}\right| \exp \left(-\int_{t_{0}}^{t} x_{i} d t\right)$. To estimate the values of $x_{i}$ below the following transformations and comparison must be made

$$
\begin{aligned}
& \frac{d}{d t}\left(\frac{x_{i}}{1-x_{i}}\right)=\frac{\dot{x}_{i}\left(1-x_{i}\right)-\left(-\dot{x}_{i}\right) x_{i}}{\left(1-x_{i}\right)^{2}}= \\
& =\frac{\left(\frac{a_{i}}{t} x_{i}-x_{i} \sum_{j=1}^{n} \frac{a_{j}}{t} x_{j}\right)\left(1-x_{i}\right)+\left(\frac{a_{i}}{t} x_{i}-x_{i} \sum_{j=1}^{n} \frac{a_{j}}{t} x_{j}\right) x_{i}}{\left(1-x_{i}\right)^{2}}=\frac{x_{i}}{1-x_{i}} \cdot \frac{a_{i}-\sum_{j=1}^{n} a_{j} x_{j}}{t\left(1-x_{i}\right)} ; \\
& \frac{1}{x_{i}}=1+\left(\frac{1}{x_{i}\left(t_{0}\right)}-1\right) \exp \left(-\int_{t_{0}}^{t} \frac{a_{i}-\sum_{j=1}^{n} a_{j} x_{j}}{t\left(1-x_{i}\right)} d t\right) \\
& -\int_{t_{0}}^{t} \frac{a_{i}-\sum_{j=1}^{n} a_{j} x_{j}}{t\left(1-x_{i}\right)} d t=-\int_{t_{0}}^{t} \frac{\sum_{j=1}^{n} a_{i} x_{j}-\sum_{j=1}^{n} a_{j} x_{j}}{t\left(1-x_{i}\right)} d t=-\int_{t_{0}}^{t} \frac{\sum_{j=1}^{n}\left(a_{i}-a_{j}\right) x_{j}}{t\left(1-x_{i}\right)} d t< \\
& <\int_{t_{0}}^{t} \frac{\sum_{j=1}^{n}\left|a_{j}-a_{i}\right| x_{j}}{t\left(1-x_{i}\right)} d t<(1-\varepsilon) \int_{t_{0}}^{t} \frac{\sum_{j \neq i} x_{j}}{t\left(1-x_{i}\right)} d t=(1-\varepsilon) \int_{t_{0}}^{t} \frac{d t}{t}=(1-\varepsilon) \ln \frac{t}{t_{0}} .
\end{aligned}
$$

These relations are valid because the inequalities (A-3) and equality $\sum_{j \neq i} x_{j}=1-x_{i}$ hold. Thus, we have that

$$
\frac{1}{x_{i}}<1+\left(\frac{1}{x_{i}\left(t_{0}\right)}-1\right) e^{(1-\varepsilon) \ln \frac{t}{t_{0}}}=1+\left(\frac{1}{x_{i}\left(t_{0}\right)}-1\right)\left(\frac{t}{t_{0}}\right)^{1-\varepsilon}
$$


Basing on $x_{i}>\left(1+\left(\frac{1}{x_{i}\left(t_{0}\right)}-1\right)\left(\frac{t}{t_{0}}\right)^{1-\varepsilon}\right)^{-1}$, derive

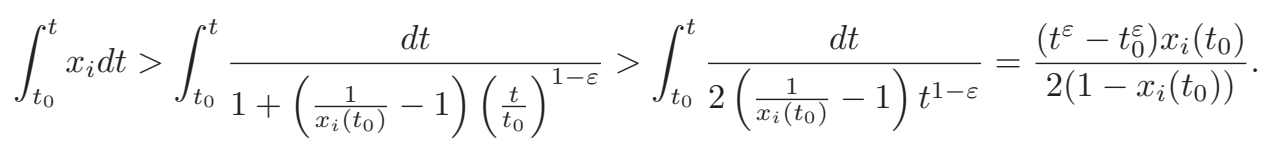

Then the relation $\left|b_{i}-a_{i}\right|=\left|b_{i}-a_{i}^{0}\right| \exp \left(-\int_{t_{0}}^{t} x_{i} d t\right)<\left|b_{i}-a_{i}^{0}\right| \exp \left(-\frac{x_{i}\left(t_{0}\right)}{2\left(1-x_{i}\left(t_{0}\right)\right)}\left(t^{\varepsilon}-t_{0}^{\varepsilon}\right)\right)$ holds. Since $\lim _{t \rightarrow \infty} \exp \left(-\frac{x_{i}\left(t_{0}\right)}{2\left(1-x_{i}\left(t_{0}\right)\right)}\left(t^{\varepsilon}-t_{0}^{\varepsilon}\right)\right)=0, \lim _{t \rightarrow \infty}\left|b_{i}-a_{i}(t)\right|=0$. Hence, $\lim _{t \rightarrow \infty} a_{i}=b_{i}$, and inequalities

$$
\int_{T}^{t} \frac{a_{i}-a_{j}}{\tau} d \tau>\int_{T}^{t} \frac{b_{i}-b_{j}-\varepsilon}{\tau} d \tau=\left(b_{i}-b_{j}-\varepsilon\right) \ln \frac{t}{T}, \quad j=\overline{1, n}, \quad j \neq i
$$

are true for any arbitrarily small $\varepsilon$ at some point $T$. Leaning on $b_{i}>\max _{j=1, n, j \neq i}\left\{b_{j}\right\}$, infer relations $(\mathrm{A}-1)$.

Acknowledgements. The reported study was partially supported by RFBR, research project No. 13-01-12452 ofi_m2.

\section{References}

[1] A. C. Allison. Protection Afforded by Sickle-cell Trait Against Subtertian Malarial Infection. Br. Med. J., 1 (1954), 290-294.

[2] R. R. Baker. The Evolutionary Ecology of Animal Migration. Holmes and Meier, New York, 1978.

[3] A. D. Bazykin. Nonlinear Dynamics of Interacting Populations. World Scientific publishing, Singapore, 1998.

[4] I. M. Bomze. Lotka-Volterra equations and replicator dynamics: a two dimensional classification. Biol. Cybernetics, 48 (1983), 201-211.

[5] A. S. Bratus, A. S. Novozhilov, A. P. Platonov. Dynamical Systems and Models in Biology. FizmMatLit, Moscow, 2010 [in Russian].

[6] A. S. Bratus, V. P. Posvyanskii, A. S. Novozhilov. A note on the replicator equation with explicit space and global regulation. Mathematical Biosciences and Engineering (MBE), 8(3) (2011), 659-676.

[7] D. S. Chernavskii. Synergetics and Information. Dynamic Information Theory. Editorial URSS, Minsk, 2004 [in Russian].

[8] D. S. Chernavskii, N. M. Chernavskaya. Some theoretical aspects of the problem of life origin. J Theor Biol., 50(1) (1975), 13-23.

[9] C. W. Clark, M. Mangel. Dynamic State Variable Models in Ecology: Methods and Applications. Oxford Series in Ecology and Evolution. Oxford University Press, 2000.

[10] R. Cressman. Evolutionary Dynamics and Existence Form Games. MIT Press, Cambridge, 2003.

[11] Ch. Darwin. On the origin of species by means of natural selection, or preservation of favoured races in the struggle for life. A Facsimile of the First Edition. Harvard University Press, Cambridge MA, 1964.

[12] R. Dawkins. Universal Darwinism. In: Evolution from molecules to man, ed. D. S. Bendall. Cambridge University Press, 1983.

[13] P. J. De Coursey. Biological Rhythms in the Marine Environment. Univ. South Carolina Press, Columbia, SC, 1976.

[14] M. Eigen. Self-Organization of Matter and the Evolution of Biological Macromolecules. Naturwissenschaften, 58 (1971), $465-523$.

[15] R. A. Fisher. The Genetical Theory of Natural Selection: A Complete Variorum Edition. Oxford: Oxford University Press, 1999.

[16] P. Fluri, R. Frick Honey bee losses during mowing of flowering fields. Bee world, 83(2002), 109-118.

[17] A. Gabriel, J. Przybylski. Sickle-cell anemia: A Look at Global Haplotype Distribution. Nature Education, 3(3):2 (2010). Available online at http://www.nature.com/scitable/topicpage/sickle-cell-anemia-a-look-at-global-8756219

[18] A. N. Gorban. Equilibrium encircling. Equations of chemical kinetics and their thermodynamic analysis. Nauka, Novosibirsk, 1984 [in Russian].

[19] A. N. Gorban. Selection Theorem for Systems with Inheritance. Math. Model. Nat. Phenom., 2(4) (2007), 1-45.

[20] A. N. Gorban, R. G. Khlebopros. Demon of Darwin: Idea of optimality and natural selection. Nauka (FizMatGiz), Moscow, 1988 [in Russian].

[21] J. B. S. Haldane. The Causes of Evolution. Princeton Science Library, Princeton University Press, 1990. 
[22] M. A. Hanin. Energy and optimality criteria developmental processes. in Mathematical Biology development, 177-187, Nauka, Moscow, 1982 [in Russian].

[23] M. A. Hanin, N. L. Dorfman. Natural selection and extremum principle. in Thermodynamics and kinetics of biological process. Nauka, Moscow, 1980 [in Russian].

[24] J. Hofbauer, K. Sigmund. Evolutionary game dynamics. Bull. (New Series) American Math. Soc. 40(4) (2003), $479-519$.

[25] G. E. Insarov. Stepped model of growth and reproduction of organisms. in Quantitative aspects of the growth of organisms. Nauka, Moscow, 1975 [in Russian].

[26] G. P. Karev. On mathematical theory of selection: Continuous time population dynamics. J. of Mathematical Biology, 60 (2010), 107-129.

[27] G. P. Karev. Replicator equations and the principle of minimal production of information. Bull Math Biol, 72(5) (2010), 1124-1142.

[28] G. P. Karev, I. G. Kareva. Replicator Equations and Models of Biological Populations and Communities. Math. Model. Nat. Phenom., 9(3) (2014), 68-95.

[29] G. P. Karev, A. S. Novozhilov, F. S. Berezovskaya. On the asymptotic behavior of the solutions to the replicator equation. Math Med Biol, 28(2) (2011), 89-110.

[30] A. Y. Klimenko. Entropy and equilibria in competitive systems. Entropy, 16 (2014), 1-22.

[31] O. A. Kuzenkov. Mathematical modelling selection processes. Mathemtical modelling and optimal control, Lobachevsky State University: Nizhnii Novgorod (1994), 120-131 [in Russian].

[32] O. A. Kuzenkov. A dynamical system on the set of Radon probability measures. Differential Equations, 31(1995), 549-554.

[33] O. A. Kuzenkov. An optimal control for a Volterra distributed system. Automation and Remote Control, 67(7) (2006), 1028-1038.

[34] O. A. Kuzenkov. The investigation of the population dynamics control problems based on the generalized Kolmogorov model. Journal of Computer and Systems Sciences International, 48(5) (2009), 839-846.

[35] O. A. Kuzenkov, G. V. Kuzenkova. Optimal control of self-reproduction systems. Journal of Computer and Systems Sciences International, 51(4) (2012), 500-511.

[36] O. A. Kuzenkov, E. A. Ryabova, K. R. Krupoderova. Mathematical models of selection processes. Lobachevsky State University: Nizhni Novgorod, 2012 [in Russian]. Available online at http://www.unn.ru/books/met_files/kuzryab.pdf

[37] O. A. Kuzenkov, E. A. Ryabova. Mathematical modeling of selection processes. Lobachevsky State University: Nizhnii Novgorod, 2007 [in Russian].

[38] T. R. Malthus. An essay on the principal of Population. Penguin Books, 1985.

[39] J. Mylius, O. Diekmann. On evolutionary stable life histories, optimization and the need to be specific about density dependence. OIKOS 74, 218-224, Copenhagen, 1995.

[40] D. W. Narver. Diel vertical movements and feeding of underyearling sockeye salmon and the limnetic zooplankton in Babine Lake, British Columbia. J. Fish. Res. Board Can, 27 (1970), 281-316.

[41] J. Neumann. Theory of Self-Reproducing Automata. Univ. of Illinois Press, Urbana, 1966.

[42] G. Parker, J. M. Smith. Optimality theory in evolutionary biology. Nature, 348 (1990), 27-33.

[43] K. Parvinen. Evolutionary suicide. Acta Biotheoretica, 53(3) (2005), 241-264.

[44] K. Parvinen. Metapopulation dynamics and the evolution of sperm parasitism. Math. Model. Nat. Phenom., 9(3) (2014), $124-137$.

[45] Y. A. Pykh. Equilibrium and stability in models of population dynamics. Nauka, Moscow, 1983 [in Russian].

[46] P. Racsko, M. Semenov. Analysis of mathematical principles in crop growth simulation models. Ecological Modelling, 47 (1989), 291-302.

[47] M. Romanovsky, N. Stepanov, D. Chernavskii. Mathematical modeling in biophysics. Nauka, Moscow, 1975 [in Russian].

[48] R. Rosen. Optimality Principles in Biology. Butterworths, London, 1967.

[49] L. I. Rozonoer and E. I. Sedyh. On the mechanisms of of evolution of selfreproduction systems. Automation and Remote Control, 40 (1979), 243-251; 419-429; 741-749.

[50] F. N. Semevsky and S. M. Semenov. Mathematical modeling of ecological processes. Gidrometeoizdat, Leningrad, 1982 [in Russian].

[51] J. M. Smith. Evolution and the theory of games. Cambridge Univ. Press, Cambridge, 1982.

[52] Y. M. Svirezhev. Phenomenological thermodynamics of interacting populations. J. of General Biology, 52(6) (1991), 840-853 [in Russian].

[53] Y. M. Svirezhev, D. O. Logofet. Stability of biological communities. Nauka, Moscow, 1978 [in Russian].

[54] P. D. Taylor, L. B. Jonker. Evolutionary stable strategies and game dynamics. Math. Biosci., 40 (1978), $145-156$.

[55] A. T. Teriokhin. Evolutionarily optimal age schedule of repair: Computer modeling of energy allocation between current and future survival and reproduction. Evolutionary Ecology, 12 (1998), 291-307.

[56] A. T. Teriokhin. Optimization modeling the evolution of the life cycle. Dissertation for the degree of Doctor of Biological Sciences. Lomonosov Moscow State University, Moscow, 2001 [in Russian].

[57] P. F. Verhulst. Notice sur la loi que la population suit dans son accroissement. Corr. Math. et Phys., 10 (1838), $113-121$

[58] V. Volterra. Lecons sur la Theorie Mathematique de la Lutte pour la Vie. Paris, 1931

[59] S. Wright. Evolution: Selected Papers. University of Chicago Press, Chicago, 1986.

[60] L. C. Young. Lectures on the Calculus of Variations and Optimal Control Theory. Philadelphia, 1969. 
[61] T. M. Zaret. Predation and Freshwater Communities. Yale Univ. Press, New Haven, CT, 1980.

[62] E. C. Zeeman. Population dynamics from game theory. in Global theory of dynamical systems. Lecture notes in mathematics. vol. 819. Springer Berlin Heidelberg, 1980.

[63] B. Zeide. Quality as a characteristic of ecological models. Ecological Modelling, 55 (1991), 161-174. 